\title{
IDENTIFICATION OF LINES IN THE SPECTRUM OF THE QUASAR $0237-233$
}

\author{
Y. P. VARSHNI \\ Department of Physics, University of Ottawa, Ottawa, Canada
}

(Received 16 May, 1988)

\begin{abstract}
The emission and absorption lines $(\lambda 23716-4290)$ in the spectrum of the quasar 0237-233 are identified within the framework of the PLS model. The available evidence indicates that it is a helium star. Similarities between the spectral properties of $0237-233$ and the star Upsilon Sagittarii are pointed out. Predictions are made for the absorption-line spectrum which falls outside $\lambda 23716-4290$ and also for an expected discontinuity at $\lambda 2600$.
\end{abstract}

\section{Introduction}

The object PKS 0237 - 23 was identified as a quasar by Arp et al. (1967). They reported five emission lines in its spectrum and interpreted the spectrum on the redshift hypothesis as having an apparent redshift of $\simeq 2.22$. This quasar has played an important role in the history of quasars. So many absorption lines were found in the spectrum of this quasar that multiple redshift hypothesis was advanced to account for them. In this paper we identify the emission and absorption lines in the spectrum of this quasar on the basis of the plasma-laser star (PLS) model of quasars (Varshni, 1973, 1974, 1975, 1977a, b, 1978, 1979, 1985, 1988a, b; Varshni and Lam, 1976; Varshni and Nasser, 1986). Our theory does away with the artificial assumption of redshifts. Laser action is responsible for the broad emission lines.

\section{Emission Lines}

\subsection{THE DATA}

There are several other quasars which belong to the same spectral class (Varshni, 1976) as $0237-233$. We summarize the available data on emission lines for all these quasars in Table I. There are a number of other quasars (Hewitt and Burbidge, 1987) which appear to belong to this spectral group but the available data are too poor to make a definite assignment. For each quasar the first row shows the reported wavelengths. Below each wavelength, where known, are shown the characteristics of that line. For three of these quasars, $0237-233,0029+003$, and $1448-232$ the available emission line data appear to be better than those for the rest.

$0237-233$. There are only two sets of measurements of the emission lines, one due to Arp et al. (1967) and the other due to Burbidge (1967). Arp et al. (1967) obtained the spectra (for emission lines) at a dispersion of $600 \AA \mathrm{mm}^{-1}$ at the prime-focus of the Lick 120 -inch telescope. These authors found five emission lines. Burbidge (1967) also used the Lick 120-inch telescope and the spectrum was obtained at a dispersion of 
$370 \AA \mathrm{mm}^{-1}$ on baked IIaO emulsion. She found two emission lines. There are large differences in the wavelengths of these two lines as given by Arp et al. (1967) and by Burbidge (1967).

$0029+003$. This quasar is one of a close pair of quasars. The other member is $0028+003$; the separation between the two is $62^{\prime \prime}$. Shaver et al. (1982) observed the two quasars at low $\left(114 \AA \mathrm{mm}^{-1}\right)$ and intermediate $\left(59 \AA \mathrm{mm}^{-1}\right)$ dispersion with the European Southern Observatory $3.6 \mathrm{~m}$ telescope, and at high dispersion $\left(33 \AA \mathrm{mm}^{-1}\right)$ with the Anglo-Australian telescope in October 1981. The line at $\lambda 3920$ is absent. For other quasars in Table $I$, in the redshift hypothesis, this line has been identified with $L \alpha$. Its absence in $0029+003$ shows that such redshifts which primarily depend on a line being identified as $\mathrm{L} \alpha$ are highly suspect.

1448 - 232. The best data appear to be that of Chen et al. $(1983,1984)$. Their observations were carried out in 1977, 1979, and 1980 at a dispersion of about $33 \AA \mathrm{mm}^{-1}$ with the Anglo-Australian telescope.

\subsection{LINE IDENTIFICATIONS}

Line No. 1. - Arp et al. (1967) give the wavelength as $3930 \AA$ A, while Burbidge (1967) gives $3919.8 \AA$. Arp et al. note that their measurement of the 23930 line was affected by the presence of the mercury night-sky line at $\lambda 4046$. The better dispersion used by Burbidge (1967) as well as the tracings given in Bahcall et al. (1968; hereafter referred to as BGS) favouring Burbidge's value. We identify this line with CII $\lambda \lambda 3919,3920.7$ (mult. 4) which is also known to occur in WR spectra and in nova like stars.

Line No. 2. - There is a considerable spread in the reported wavelengths. Assuming that the one reported by Shaver et al. (1982) is closest to the correct value, the identification is with N II $\lambda 3995$ (mult. 12). The line occurs in Wolf-Rayet and novae spectra.

It is of interest to compare the behaviour of $0237-233$ and $0029+003$ with respect to Line Nos. 1 and 2. In 0237 - 233, No. 1 occurs but No. 2 does not, while in $0029+03$, the reverse is the case. Precisely this type of behaviour is expected from laser action in our theory (Varshni, 1975, 1979) for two quasars which lie at certain positions on the $n_{e}, T_{e}$ diagram. The redshift hypothesis has no explanation for this behaviour.

Line No. 3. - The recorded values for the wavelength are $4537 \AA$ (Arp et al., 1967) and $4513 \AA$ (Burbidge, 1967). As Burbidge used a better dispersion her value appears to be more accurate. We identify this line with $\mathrm{C}_{\mathrm{III}} \lambda \lambda 4516,4517$ (mult. 9). This line occurs with good strength in WC spectra. We would like to mention here another possibility also - N III $\lambda \lambda 4511,4515,4518$ (mult. 3). This line is often quite strong in WN spectra. A detailed study of the structure of the quasar line can help to decide which of the two is the correct identification.

Line No. 4. - We identify this line with N II $\lambda \lambda 4987,4994$ (mult. 24).

Line No. 5. - It appears only as a weak line and its wavelength has considerable uncertainty. We identify this line with C III $\lambda \lambda 5273,5254$ (mult. 4). This line appears in WC spectra.

Line No. 6. - For this line also, there is considerable uncertainty in its wavelength, Arp et al. (1967) note that their measurement of $\lambda 6141$ was affected by the night-sky 
TABLE I

Emission line data for quasars. Below each wavelength are given, where available, the characteristics of the line. The symbols have the following meanings. Br broad; $\mathrm{M}$ - medium strength; S - strong; Wk - weak; VWk - very weak. For 0029 + 003, 0046 - 297, and 2228.6 - 395, the second line shows the equivalent widths. For $0244-128,0851+197$, and $1448-132$, in the second line, the values in decimals indicate the line to continuum ratios, and the values in $\AA$ are the linewidths.

\begin{tabular}{|c|c|c|c|c|c|c|c|c|c|}
\hline Quasar & $\begin{array}{l}V \\
\text { (Mag) }\end{array}$ & Set & $\begin{array}{l}1 \\
\lambda(\AA)\end{array}$ & $\begin{array}{l}2 \\
\lambda(\AA)\end{array}$ & $\begin{array}{l}3 \\
\lambda(\AA)\end{array}$ & $\begin{array}{l}4 \\
\lambda(\AA)\end{array}$ & $\begin{array}{l}5 \\
\lambda(\AA)\end{array}$ & $\begin{array}{l}6 \\
\lambda(\AA)\end{array}$ & Source \\
\hline \multirow[t]{3}{*}{$0237-233$} & 16.63 & (a) & 3930 & & 4537 & 4993 & 5285 & 6141 & Arp et al. (1967) \\
\hline & & & $\mathrm{S}$ & & M & $\mathrm{S}$ & Wk & $\mathbf{M}$ & \\
\hline & & (b) & 3919.8 & & 4513.7 & & & & Burbidge (1967) \\
\hline \multirow[t]{2}{*}{$0029+003$} & 18 & & absent & 3994 & 4507 & 4991 & & 6160 & Shaver et al. (1982) \\
\hline & & & & $160 \AA$ & $21 \AA$ & $73 \AA$ & & $36 \AA$ & \\
\hline \multirow[t]{2}{*}{$0046-297$} & 19.5 & & 3909 & & & 4964 & & & Savage et al. (1985) \\
\hline & & & $200 \AA$ & & & $60 \AA$ & & & \\
\hline \multirow[t]{2}{*}{$0135-002$} & 19.1 & & 3900 & & & 5000 & & & MacAlpine and Lewis (1978) \\
\hline & & & $\mathrm{M}, \mathrm{Br}$ & & & VWk & & & \\
\hline \multirow[t]{2}{*}{$0242-410$} & 18.1 & & 3905 & 3984 & & 4986 & 5267 & 6155 & Osmer and Smith (1977) \\
\hline & & & $\mathrm{S}$ & Wk & & $\mathbf{M}$ & Wk & $\mathrm{Wk}$ & \\
\hline \multirow[t]{2}{*}{$0244-128$} & 17.1 & & 3896 & (3989) & 4480 & 4952 & 5253 & 6120 & Jauncey et al. (1984) \\
\hline & & & $4.46,70 \AA$ & 0.6 & $0.5,50 \AA$ & $2.07,50 \AA$ & $0.90,15 \AA$ & $1.0,65 \AA$ & \\
\hline $0254-671$ & 18.5 & & & 3970 & & 4995 & & & Savage et Wright (1981) \\
\hline \multirow{2}{*}{$0851+197$} & 18 & & 3912 & & 4494 & 4972 & & & Schmidt (1974) \\
\hline & & & $S, 150 \AA$ & & Wk & $\mathrm{Wk}$ & & & \\
\hline \multirow[t]{2}{*}{$1225+317$} & 15.87 & & 3930 & & & 4950 & & & Ulrich (1976) \\
\hline & & & $\mathrm{S}$ & & & Wk & & & \\
\hline \multirow[t]{2}{*}{$1246-057$} & 16.73 & & & 3976 & & 4983 & & & Osmer and Smith (1977) \\
\hline & & & & $\mathrm{S}$ & & $\mathbf{M}$ & & $\cdot$ & \\
\hline \multirow[t]{5}{*}{$1448-232$} & 16.96 & (a) & 3900 & 3980 & 4480 & 4970 & & 6125 & Savage et al. (1976) \\
\hline & & & $2.5,60 \AA$ & 1.0 & 0.25 & $1.0,45 \AA$ & & $0.4,120 \AA$ & \\
\hline & & (b) & 3919 & & 4494 & 4985 & & & Osmer and Smith (1977) \\
\hline & & & $\mathrm{S}$ & & Wk & $\mathbf{M}$ & & & \\
\hline & & (c) & 3911 & 3985 & 4515 & $(4981)$ & & & Chen et al. $(1983,1984)$ \\
\hline $1816+475$ & 18.2 & & 3928 & & 4508 & 4986 & & & Walsh et al. (1979) \\
\hline $2228.6-395$ & 18.3 & & 3895 & & 4479 & 4971 & & 6094 & Savage et al. (1985) \\
\hline & & & $340 \AA$ & & & & & & \\
\hline
\end{tabular}


line at $\lambda 6300$. We identify this line with $\mathrm{C}$ III $\lambda \lambda 6154,6155,6157$ (mult. 13). This line also appears in WC spectra.

The proposed identifications depend, of course, on the assumption that the reported wavelengths are correct. Should future investigations show that the actual values of the wavelengths are substantially different from those given by Burbidge (1967) and Shaver et al. (1982), clearly the question of identification will have to be reconsidered.

\section{Absorption-Line Spectrum}

We have discussed in previous papers (Varshni, 1977, 1978, 1985) the characteristics of the absorption-line spectrum of quasars that are predicted from our model. In particular we shall follow the considerations of our 1985 paper.

There have been a number of investigations on the absorption-line spectrum of 0237 - 233. We summarize these investigations here.

A spectrum of $195 \AA \mathrm{mm}^{-1}$ obtained by Arp et al. (1967) at the prime focus of the 200 -inch telescope showed the presence of many absorption lines. These authors listed 33 absorption lines in the interval $\lambda \lambda 3526-5096$ and attempted to identify them on the redshift hypothesis with $z=2.20$. Burbidge (1967) obtained a spectrum of $0237-23$ on baked IIaO emulsion with the conventional prime-focus spectrograph on the Lick 120-inch telescope, with the camera-grating combination giving a dispersion of $370 \AA \mathrm{mm}^{-1}$. She listed 20 lines in the interval $\lambda \lambda 3444-4613$ and attempted to identify some of the lines at $z \simeq 1.95$. Greenstein (cf. Greenstein and Schmidt, 1967) obtained two spectra of $0237-233$ at a higher resolution. They were obtained at $89 \AA \mathrm{mm}^{-1}$ in the first order with the Palomar prime-focus spectrograph. About 150 possible absorption features were measured. Of these, as described by Greenstein and Schmidt (1967), a highly selected list of 40 lines was published in their Table I. It is not clear how many strong lines were left unmentioned. Greenstein and Schmidt (1967) assigned most of these 40 lines to two redshift systems, $z_{1}=2.2020$ and $z_{2}=1.9555$. Thus was born the multiple-redshift hypothesis for the absorption lines of quasars.

Meanwhile several more spectrograms were obtained by Greenstein and Sargent (BGS) and a list of 49 absorption lines in the range $\lambda \lambda 3346-4493$ was analyzed in collaboration with Bahcall using the multiple-redshift hypothesis. Twenty-eight of the forty-nine lines were claimed to have been identified by five redshifts. These were $z=2.2015,1.6704,1.6560,1.5132$, and 1.3642 . However, many of the strong lines were unidentified, even with five redshift systems. It is not without significance that after this work on 0237 - 233, Greenstein, a very experienced stellar spectroscopist, quit quasars altogether and years later wrote 'as for me, after a few parting shots on multipleabsorption-line systems in quasars, I abandoned the field in 1967 without regret' (Greenstein, 1984). Parenthetically we note that it was not for the lack of telescope time, because as he himself notes, he had been assigned 20 to 30 nights a year (1952-1979) at the 200-inch (Greenstein, 1984). Greenstein's work on a similar star is noted in Section 5.

Burbidge et al. (1968; hereafter BLS) obtained spectra of 0237 - 233 at Lick and Kitt 
Peak observatories and they list a total of 92 absorption lines in the interval $\lambda \lambda 3205-6630$. Comparing their results with those of Greenstein and Schmidt (1967), Burbidge et al. commented: "However, it is clear that the present material does not support a contention that the majority of the absorption features in PKS 0237-23 can be explained on the basis of the two suggested redshifts. Furthermore, the present authors take issue with many of the identifications made by Greenstein and Schmidt...". BLS proposed an additional redshift, $z=1.365$. Grueff (1969) claimed that eleven absorption lines of strong and medium intensity can be identified with lines of sulphur ions at a redshift of 2.4516 .

The investigations of BLS and BGS were based on conventional image-tube and photographic spectrograms which were near the limits of the resolution, about $2.3 \AA$, then available on large telescopes.

Boksenberg and Sargent (1975) used the University College London image photon counting system (IPCS) at the Coudé focus of the Palomar telescope to obtain the spectrum of $0237-233$ at a resolution of $0.71 \AA$. They give a list of 75 absorption lines in the wavelength range $\lambda \lambda 3737-4270$. Boroson et al. $(1978$, hereafter BSBC) carried out further observations on $0233-233$ with the IPCS and these observations were combined with the older data of Boksenberg and Sargent (1975). These authors tabulated wavelengths and equivalent widths of 193 absorption lines in the spectral range $\lambda \lambda 3716-4289$. BSBC claimed that many of these lines are due to C IV $\lambda \lambda 1548.20$, 1550.77 at various redshifts. A total of 45 redshifts were claimed. Varshni (1981) examined these supposed identifications and showed that the number of redshift systems based on CIV doublets differs insignificantly from what would be expected from chance coincidences. Consequently, these systems and their $z$-values seem devoid of any physical significance.

Varshalovich and Levshakov (1981) analyzed the absorption line data reported by BSBC and claimed to have found 12 redshift systems containing CO lines and they tabulate five of these. Varshni (1983) examined this claim also and showed that the number of redshifted $\mathrm{CO}$ absorption-line systems that are found in the spectrum of 0237 - 233, using the rules of Varshalovich and Levshakov, differs insignificantly from what would be expected from chance coincidences. Consequently, the CO systems proposed by Varshalovich and Levshakov also seem to have no physical reality.

It is obvious from the above summary that the redshift hypothesis has been singularly unsuccessful in explaining the absorption-line spectrum of $0237-233$. Before coming to the identifications on the basis of our theory, we shall first consider the quality of the available data. Certain problems whih were encountered in the case of $0805+046$ (Varshni, 1985) are also present here.

With the improvement in observational techniques, the number of observed lines has been rapidly increasing. This can be seen in the number density of lines observed. Most of the reported observations of 0237 - 233 are shortward of $5100 \AA$, and in this region the number of lines per $100 \AA$ for the various investigators are as follows: Arp et al. (1967) - 2.10, BLS (1968) - 4.51 (Kitt Peak spectrum) and 4.45 (Lick spectrum), BGS (1968) - 4.27, Boksenberg and Sargent (1978) - 13.70, BSBC (1978) - 33.68. The 
resolution obtained by BSBC was only $0.7 \AA$ (corresponds to $52 \mathrm{~km} \mathrm{~s}^{-1}$ ). Now it is possible to obtain much better resolution in quasar spectra (Chaffee et al., 1983, $12 \mathrm{~km} \mathrm{~s}^{-1}$; York et al., 1984, $10 \mathrm{~km} \mathrm{~s}^{-1}$ ). If $0237-233$ is studied with a resolution of $10 \mathrm{~km} \mathrm{~s}^{-1}$, we expect the number density of lines to increase by a factor of about 2.5. The incompleteness of the BSBC data has to be borne in mind while considering the number of lines of any element which are identified as against the expected number of lines, and also as regards the completeness of multiplets in the identifications.

As noted earlier, the estimated resolution in the BSBC data is $0.71 \AA$. However, a close inspection of enlargements of Figures 1 and 2 of BSBC shows that many lines are wider than $0.7 \AA$ and many of them are blends. BSBC state "The wavelengths of the lines are probably accurate to $0.3 \AA$ except in the case of blended lines, where the error may be twice as large". Allowing for these various factors a value of $1 \AA$ appears to be a reasonable guiding value for the tolerance: i.e., the discrepancy between the observed and identified wavelengths. When the line is very wide (blended) clearly a larger value is acceptable.

As regards the relative intensities of lines, again the situation is similar to that of $0805+046$. We would give here only two examples. For the lines $\lambda 3886.70$ and $\lambda 3891.8$, Boksenberg and Sargent (1975) give the intensities as 3 and 5, respectively, while BSBC give 1.9 and 0.9 , respectively. Similarly for $\lambda 3905.5$ and 23909.8 , Boksenberg and Sargent's (1975) values are 3 and 2, while BSBC give 0.9 and 0.1 , respectively. Clearly the recorded equivalent widths can only be taken as a qualitative guide and no more.

Identifications for most of the absorption lines in the range $\lambda \lambda 3716-4290$ reported by BSBC are presented in Table II. The reported equivalent widths of lines by BSBC appear to indicate that in some cases, besides the proposed identifications, there is blending due to unknown components. The average value of $\left|\lambda_{\text {obs }}-\lambda_{\text {iden }}\right|$ for all the identifications is $0.49 \AA$ which is quite satisfactory. Next we consider the evidence bearing on the presence of some of the atoms and ions.

He I. Present. Mult. 2, 5, 16, and 18 are present. The only line missing is $\lambda 3868$, which could be weak, or blended with $\lambda 3869.70$. The weakness of $\lambda 3889$ is rather surprising.

Mg I. Appears to be present though weakly.

SiII. Present. Multiplets 1 and 3 are present, which are the only important ones expected.

Ca II. Present. Part of the equivalent widths of $\mathrm{K}$ and $\mathrm{H}$ lines is expected to be interstellar.

Sc II. Possibly represented weakly by the line $\lambda 4247$.

TiII. Present. Most members of multiplets 11, 13, 34, 72, and 105 are present.

CrII. Represented by many lines.

Fe I. Many lines are present.

Fe II. Represented by many lines.

Sr II. Multiplet 1 is present.

There is no positive evidence for the presence of hydrogen in the available data. Clearly the quasar is highly deficient in hydrogen. It will be noticed that most of the lines which we have not been able to identify are common with unidentified lines in the star $v$ Sgr. 
TABLE II

Identification of lines in the spectrum of $0237-233 . \delta=\lambda_{\text {obs }}-\lambda_{\text {lab }} \cdot \lambda_{\text {obs }}, W_{\lambda}, \lambda_{\text {lab }}$, and $\delta$ all are in $\AA$.

\begin{tabular}{|c|c|c|c|c|c|}
\hline \multirow[t]{2}{*}{$\lambda_{\text {obs }}$} & \multirow[t]{2}{*}{$W_{\lambda}$} & \multicolumn{3}{|c|}{ Identification } & \multirow[t]{2}{*}{$\delta$} \\
\hline & & $\lambda_{\text {lab }}$ & Ion & Mult. & \\
\hline \multirow[t]{2}{*}{3716.90} & \multirow[t]{2}{*}{0.6} & 3715.45 & CrII & 145 & 1.45 \\
\hline & & 3717.53 & Mn II & 8 & -0.63 \\
\hline 3721.70 & 0.3 & 3721.63 & Ti II & 13 & 0.07 \\
\hline \multirow[t]{2}{*}{3722.80} & \multirow[t]{2}{*}{0.9} & 3722.56 & $\mathrm{Fe} I$ & 5 & 0.24 \\
\hline & & 3723.40 & Cr II & 144 & -0.60 \\
\hline \multirow[t]{4}{*}{3726.50} & \multirow[t]{4}{*}{0.9} & 3727.04 & Fe II & 192 & -0.54 \\
\hline & & 3727.35 & V II & 21 & -0.85 \\
\hline & & 3727.37 & CrII & 117 & -0.87 \\
\hline & & 3727.62 & $\mathrm{Fe} I$ & 21 & -1.12 \\
\hline \multirow[t]{3}{*}{3737.80} & \multirow[t]{3}{*}{0.5} & 3736.90 & Ca II & 3 & 0.90 \\
\hline & & 3737.13 & $\mathrm{Fe}_{\mathrm{I}}$ & 5 & 0.67 \\
\hline & & 3738.38 & CrII & 20 & -0.58 \\
\hline 3739.40 & 0.6 & 3739.53 & $\mathrm{Fe}_{\mathrm{I}}$ & & -0.13 \\
\hline 3741.90 & 0.2 & 3741.63 & TiII & 72 & 0.27 \\
\hline 3742.60 & 0.3 & 3742.64 & v Sag & & \\
\hline 3743.30 & 0.5 & 3743.36 & $\mathrm{Fe} I$ & 21 & -0.06 \\
\hline \multirow[t]{4}{*}{3746.00} & \multirow[t]{4}{*}{0.1} & 3745.56 & $\mathrm{Fe} I$ & 5 & 0.44 \\
\hline & & 3745.81 & V II & 15 & 0.19 \\
\hline & & 3745.90 & $\mathrm{Fe} I$ & 5 & 0.10 \\
\hline & & 3746.56 & Fe II & 14 & -0.56 \\
\hline 3747.40 & 1.1 & 3748.01 & TiII & 107 & -0.61 \\
\hline \multirow[t]{4}{*}{3748.80} & \multirow[t]{4}{*}{0.2} & 3748.26 & $\mathrm{Fe} I$ & 5 & 0.54 \\
\hline & & 3748.49 & $\mathrm{Fe}$ II & 154 & 0.31 \\
\hline & & 3748.50 & $\mathrm{Fe} I$ & 5 & 0.30 \\
\hline & & 3748.68 & Cr II & 11 & 0.12 \\
\hline \multirow{2}{*}{3750.30} & \multirow[t]{2}{*}{0.2} & 3750.56 & CrII & & -0.26 \\
\hline & & 3750.88 & VII & 21 & -0.58 \\
\hline 3751.60 & 0.4 & 3751.22 & V II & 100 & 0.38 \\
\hline \multirow[t]{2}{*}{3754.20} & \multirow[t]{2}{*}{0.4} & 3753.61 & $\mathrm{Fe} I$ & 73 & 0.59 \\
\hline & & 3754.59 & CrII & 20 & -0.39 \\
\hline \multirow[t]{2}{*}{3758.30} & \multirow[t]{2}{*}{0.2} & 3757.68 & TiII & 72 & 0.62 \\
\hline & & 3758.23 & $\mathrm{Fe} I$ & 21 & 0.06 \\
\hline 3759.60 & 0.8 & 3759.29 & TiII & 13 & 0.31 \\
\hline & & 3759.46 & Fe II & 154 & 0.14 \\
\hline & & 3760.24 & V II & 21 & -0.64 \\
\hline 3761.10 & 0.3 & 3761.32 & TilI & 13 & -0.22 \\
\hline & & 3761.69 & CrII & 11 & -0.59 \\
\hline & & 3761.90 & Cr II & 11 & -0.80 \\
\hline 3762.80 & 0.3 & 3762.89 & FeII & 192 & -0.09 \\
\hline 3763.80 & 0.2 & 3763.79 & $\mathrm{Fe}_{\mathrm{I}}$ & 21 & 0.01 \\
\hline & & 3764.09 & Fe II & 29 & -0.29 \\
\hline 3767.80 & 0.8 & 3767.19 & $\mathrm{Fe} I$ & 21 & 0.61 \\
\hline 3773.30 & 0.5 & 3773.66 & v Sag & & \\
\hline & & 3773.77 & $48 \mathrm{Lib}$ & & \\
\hline 3776.30 & 0.2 & 3776.06 & Ti II & 72 & 0.24 \\
\hline 3777.50 & 1.8 & 3778.36 & VII & 21 & -0.86 \\
\hline
\end{tabular}


Table II (continued)

\begin{tabular}{|c|c|c|c|c|c|}
\hline \multirow[t]{2}{*}{$\lambda_{\mathrm{obs}}$} & \multirow[t]{2}{*}{$W_{\lambda}$} & \multicolumn{3}{|c|}{ Identification } & \multirow[t]{2}{*}{$\delta$} \\
\hline & & $\lambda_{\text {lab }}$ & Ion & Mult. & \\
\hline 3777.50 & 1.8 & 3778.37 & Fe II & 192 & -0.87 \\
\hline \multirow[t]{2}{*}{3788.00} & 0.5 & 3787.23 & $\mathrm{~V}_{\text {II }}$ & 100 & 0.76 \\
\hline & & 3787.88 & $\mathrm{Fe}_{\mathrm{I}}$ & 21 & 0.12 \\
\hline 3789.20 & 0.1 & 3789.31 & Pleione & & \\
\hline \multirow[t]{2}{*}{3790.60} & 1.1 & 3790.06 & $48 \mathrm{Lib}$ & & \\
\hline & & 3791.43 & $\varepsilon$ Aurigae & & \\
\hline 3792.30 & 0.5 & 3791.41 & SiIII & 5 & 0.89 \\
\hline 3795.10 & 0.2 & 3795.00 & $\mathrm{Fe}_{\mathrm{I}}$ & 21 & 0.10 \\
\hline \multirow[t]{3}{*}{3799.20} & 0.3 & 3798.36 & Fe II & 14 & 0.84 \\
\hline & & 3799.55 & $\mathrm{Fe}_{\mathrm{I}}$ & 21 & -0.35 \\
\hline & & 3799.81 & TiII & 13 & -0.61 \\
\hline 3803.60 & 0.3 & & & & \\
\hline \multirow[t]{2}{*}{3804.70} & 0.5 & 3805.34 & $\mathrm{Fe}_{\mathrm{I}}$ & 608 & -0.65 \\
\hline & & 3805.40 & $\alpha$ Cygni & & \\
\hline \multirow[t]{2}{*}{3807.40} & 0.1 & 3806.70 & $\mathrm{Fe}_{\mathrm{I}}$ & 607 & 0.70 \\
\hline & & 3806.82 & $\mathrm{Fe}_{\mathrm{II}}$ & 153 & 0.58 \\
\hline \multirow[t]{2}{*}{3810.90} & 0.3 & 3810.41 & Pleione & & \\
\hline & & 3810.48 & $\varepsilon$ Aurigae & & \\
\hline 3811.90 & 0.1 & 3812.96 & $\mathrm{Fe}_{\mathrm{I}}$ & 22 & -1.06 \\
\hline \multirow[t]{2}{*}{3821.70} & 0.1 & 3820.43 & $\mathrm{Fe}_{\mathrm{I}}$ & 20 & 1.27 \\
\hline & & 3821.92 & Fe II & 14 & -0.22 \\
\hline \multirow[t]{3}{*}{3826.90} & 0.1 & 3825.88 & $\mathrm{Fe}_{\mathrm{I}}$ & 20 & 1.02 \\
\hline & & 3827.08 & Fe II & 153 & -0.18 \\
\hline & & 3827.82 & $\mathrm{Fe}_{\mathrm{I}}$ & 45 & -0.92 \\
\hline 3831.20 & 0.1 & 3832.30 & $\mathrm{Mg}_{\mathrm{I}}$ & 3 & -1.10 \\
\hline 3838.50 & 1.2 & 3838.29 & $\mathrm{Mg}_{\mathrm{I}}$ & 3 & 0.21 \\
\hline \multirow[t]{2}{*}{3840.00} & 0.5 & 3840.44 & $\mathrm{Fe}_{\mathrm{I}}$ & 20 & -0.44 \\
\hline & & 3841.05 & $\mathrm{Fe}_{\mathrm{I}}$ & 45 & -1.05 \\
\hline \multirow[t]{2}{*}{3842.50} & 0.1 & 3843.00 & Sc II & 1 & -0.50 \\
\hline & & 3843.03 & Zr II & 7 & -0.53 \\
\hline 3845.20 & 0.1 & 3845.18 & Fe II & 127 & 0.02 \\
\hline \multirow[t]{2}{*}{3854.40} & 0.3 & 3853.66 & Si II & 1 & 0.74 \\
\hline & & 3853.95 & Fe II & 127 & 0.45 \\
\hline \multirow[t]{2}{*}{3857.30} & 0.3 & 3856.02 & Si II & 1 & 1.28 \\
\hline & & 3857.53 & $v \mathrm{Sag}$ & & \\
\hline 3861.70 & 1.4 & 3860.92 & $\mathrm{Fe} I I$ & & 0.78 \\
\hline \multirow[t]{2}{*}{3862.60} & 0.8 & 3862.59 & Si II & 1 & 0.01 \\
\hline & & 3863.40 & Fe II & 152 & -0.80 \\
\hline \multirow[t]{2}{*}{3869.70} & 0.2 & 3869.56 & $\mathrm{Fe} I$ & 284 & 0.14 \\
\hline & & 3869.59 & $\mathrm{Fe}_{\mathrm{I}}$ & 284 & 0.11 \\
\hline \multirow[t]{2}{*}{3874.20} & 0.7 & 3873.76 & $\mathrm{Fe}_{\mathrm{I}}$ & 175 & 0.44 \\
\hline & & 3874.56 & CrII & 143 & -0.36 \\
\hline 3876.80 & 0.1 & & & & \\
\hline \multirow[t]{3}{*}{3879.10} & 0.2 & 3878.02 & $\mathrm{Fe}_{\mathrm{I}}$ & 20 & 1.08 \\
\hline & & 3878.57 & $\mathrm{Fe}_{\mathrm{I}}$ & 4 & 0.53 \\
\hline & & 3878.72 & $\mathrm{~V}_{\mathrm{II}}$ & 33 & 0.38 \\
\hline 3880.30 & 0.7 & 3879.63 & v Sag & & \\
\hline 3882.00 & 0.1 & 3881.92 & Ni II & 13 & 0.08 \\
\hline
\end{tabular}


Table II (continued)

\begin{tabular}{|c|c|c|c|c|c|}
\hline \multirow[t]{2}{*}{$\lambda_{\mathrm{obs}}$} & \multirow[t]{2}{*}{$W_{\lambda}$} & \multicolumn{3}{|c|}{ Identification } & \multirow[t]{2}{*}{$\delta$} \\
\hline & & $\lambda_{\mathrm{lab}}$ & Ion & Mult. & \\
\hline 3882.00 & 0.1 & 3882.28 & TiII & 34 & -0.28 \\
\hline 3884.30 & 0.2 & 3884.85 & V II & 33 & -0.55 \\
\hline \multirow[t]{2}{*}{3886.70} & 1.9 & 3886.28 & $\mathrm{Fe} I$ & 4 & 0.42 \\
\hline & & 3887.05 & $\mathrm{Fe}_{\mathrm{I}}$ & 20 & -0.35 \\
\hline 3889.50 & 0.1 & 3888.65 & $\mathrm{He} \mathrm{I}$ & 2 & 0.85 \\
\hline 3891.80 & 0.9 & & & & \\
\hline \multirow[t]{2}{*}{3892.20} & 1.2 & 3892.14 & Cr II & & 0.06 \\
\hline & & 3892.30 & $S_{\text {II }}$ & & -0.10 \\
\hline 3892.80 & 0.8 & 3893.39 & $\mathrm{Fe} I$ & 430 & -0.59 \\
\hline \multirow[t]{2}{*}{3894.10} & 0.4 & 3893.99 & v Sag & & \\
\hline & & 3895.00 & $\mathrm{Fe} I$ & 4 & -0.90 \\
\hline \multirow[t]{2}{*}{3899.30} & 0.3 & 3899.14 & V II & 33 & 0.16 \\
\hline & & 3899.71 & $\mathrm{Fe}_{\mathrm{I}}$ & 4 & -0.41 \\
\hline \multirow[t]{2}{*}{3900.70} & 0.3 & 3900.55 & TiII & 34 & 0.15 \\
\hline & & 3900.68 & $\mathrm{Al}$ II & 1 & 0.02 \\
\hline \multirow[t]{4}{*}{3905.50} & 0.9 & 3905.53 & $\mathrm{Si} \mathrm{I}$ & 3 & -0.03 \\
\hline & & 3905.64 & Cr II & 167 & -0.14 \\
\hline & & 3906.04 & Fe II & 173 & -0.54 \\
\hline & & 3906.48 & $\mathrm{Fe} I$ & 4 & -0.98 \\
\hline \multirow[t]{2}{*}{3909.80} & 0.1 & 3908.54 & FeII & 29 & 1.26 \\
\hline & & 3909.84 & $\mathrm{Fe} I$ & 4 & -0.04 \\
\hline 3910.80 & 0.2 & & & & \\
\hline 3911.40 & 0.2 & 3911.30 & Cr II & 129 & 0.10 \\
\hline 3912.40 & 0.2 & 3913.46 & Ti II & 34 & -1.06 \\
\hline 3917.10 & 0.2 & 3916.42 & V II & 10 & 0.68 \\
\hline 3924.60 & 0.3 & 3924.91 & v Sag & & \\
\hline \multirow[t]{2}{*}{3925.40} & 0.1 & 3925.52 & v Sag & & \\
\hline & & 3925.95 & $\mathrm{Fe} \mathrm{I}$ & 364 & -0.55 \\
\hline \multirow[t]{2}{*}{3930.80} & 0.1 & 3930.30 & $\mathrm{Fe}_{\mathrm{I}}$ & 4 & 0.50 \\
\hline & & 3930.31 & $\mathrm{Fe}$ II & 3 & 0.49 \\
\hline 3933.40 & 1.3 & 3933.66 & Ca II & 1 & -0.26 \\
\hline \multirow[t]{2}{*}{3937.20} & 0.1 & 3936.95 & Cr II & 128 & 0.25 \\
\hline & & 3938.29 & Fe II & 3 & -1.09 \\
\hline \multirow[t]{3}{*}{3944.40} & 0.1 & 3944.01 & $\mathrm{Al} \mathbf{I}$ & 1 & 0.39 \\
\hline & & 3945.11 & $\mathrm{Cr}$ II & 142 & -0.71 \\
\hline & & 3945.21 & Fe II & 3 & -0.81 \\
\hline 3949.50 & 0.7 & 3949.95 & $\mathrm{Fe}_{\mathrm{I}}$ & 72 & -0.45 \\
\hline 3950.60 & 1.0 & 3951.16 & $\mathrm{Fe}_{\mathrm{I}}$ & 661 & -0.56 \\
\hline \multirow[t]{2}{*}{3954.90} & 0.1 & 3954.30 & Si II & 7.07 & 0.60 \\
\hline & & 3954.51 & Si II & 7.07 & 0.39 \\
\hline 3955.70 & 0.1 & 3956.05 & v Sag & & \\
\hline 3957.00 & 0.1 & 3956.68 & $\mathrm{Fe} I$ & 278 & 0.32 \\
\hline \multirow[t]{2}{*}{3959.70} & 0.1 & 3959.04 & $\alpha$ Cygni & & \\
\hline & & 3959.18 & $v$ Sag & & \\
\hline \multirow[t]{2}{*}{3961.70} & 0.2 & 3960.90 & Fe II & 212 & 0.80 \\
\hline & & 3961.52 & $\mathrm{Al} \mathrm{I}$ & 1 & 0.18 \\
\hline
\end{tabular}


Table II (continued)

\begin{tabular}{|c|c|c|c|c|c|}
\hline \multirow[t]{2}{*}{$\lambda_{\text {obs }}$} & \multirow[t]{2}{*}{$W_{\lambda}$} & \multicolumn{3}{|c|}{ Identification } & \multirow[t]{2}{*}{$\delta$} \\
\hline & & $\lambda_{\text {lab }}$ & Ion & Mult. & \\
\hline \multirow[t]{2}{*}{3963.70} & \multirow[t]{2}{*}{0.2} & 3964.57 & Fe II & 29 & -0.87 \\
\hline & & 3964.73 & $\mathrm{HeI}$ & 5 & -1.03 \\
\hline 3969.40 & 0.1 & 3968.47 & Ca II & 1 & 0.93 \\
\hline 3970.60 & 0.1 & 3970.19 & $v \mathrm{Sag}$ & & \\
\hline 3971.80 & 0.1 & 3972.42 & $v \mathrm{Sag}$ & & \\
\hline 3978.90 & 0.1 & 3979.51 & CrII & 183 & -0.61 \\
\hline \multirow[t]{2}{*}{3981.30} & \multirow[t]{2}{*}{0.1} & 3981.61 & $\mathrm{Fe} I \mathrm{I}$ & 3 & -0.31 \\
\hline & & 3982.00 & TilI & 11 & -0.70 \\
\hline 3984.70 & 0.1 & 3983.96 & $\mathrm{Fe} I$ & 277 & 0.74 \\
\hline 3987.30 & 0.1 & 3987.63 & TilI & 11 & -0.33 \\
\hline 3989.40 & 0.1 & 3989.80 & V II & 32 & -0.40 \\
\hline \multirow[t]{3}{*}{3992.30} & \multirow[t]{3}{*}{0.1} & 3991.77 & SiII & & 0.53 \\
\hline & & 3992.06 & $\alpha$ Cygni & & \\
\hline & & 3992.60 & V II & 10 & -0.30 \\
\hline 3999.00 & 0.1 & 3998.98 & Zr II & 16 & 0.02 \\
\hline 4001.90 & 0.2 & 4002.07 & Fe II & 29 & -0.17 \\
\hline \multirow[t]{3}{*}{4002.90} & \multirow[t]{3}{*}{0.3} & 4002.55 & Fe II & 190 & 0.35 \\
\hline & & 4003.20 & V II & 9 & -0.30 \\
\hline & & 4003.33 & CrII & 194 & -0.43 \\
\hline 4009.90 & 0.2 & 4009.30 & TiII & 11 & 0.60 \\
\hline \multirow[t]{2}{*}{4011.40} & \multirow{2}{*}{0.2} & 4012.37 & Ti II & 11 & -0.97 \\
\hline & & 4012.50 & CrII & 183 & -1.10 \\
\hline \multirow[t]{2}{*}{4017.60} & \multirow[t]{2}{*}{0.7} & 4017.16 & $\mathrm{Fe} I$ & 527 & 0.44 \\
\hline & & 4017.96 & CrII & 166 & -0.36 \\
\hline 4018.60 & 0.4 & & & & \\
\hline 4019.80 & 1.2 & 4019.72 & $v \mathrm{Sag}$ & & \\
\hline \multirow[t]{2}{*}{4024.30} & \multirow[t]{2}{*}{0.3} & 4023.39 & V II & 32 & 0.91 \\
\hline & & 4024.55 & Fe II & 127 & -0.25 \\
\hline 4025.10 & 1.2 & 4025.14 & TiII & 11 & -0.04 \\
\hline \multirow[t]{2}{*}{4026.30} & \multirow[t]{2}{*}{0.8} & 4026.19 & $\mathrm{He} \mathrm{I}$ & 18 & 0.11 \\
\hline & & 4026.36 & $\mathrm{HeI}$ & 18 & -0.06 \\
\hline \multirow[t]{2}{*}{4029.00} & 0.1 & 4028.33 & TilI & 87 & 0.67 \\
\hline & & 4029.68 & ZrII & 41 & -0.68 \\
\hline 4030.40 & 0.1 & 4030.40 & Mn I & 2 & 0.00 \\
\hline & & 4031.46 & Fe II & 151 & -1.06 \\
\hline 4032.40 & 0.1 & 4032.95 & Fe II & 126 & -0.55 \\
\hline & & 4033.07 & Mn I & 2 & -0.67 \\
\hline 4037.60 & 0.1 & 4036.78 & V II & 9 & 0.82 \\
\hline 4038.40 & 0.1 & 4038.03 & CrII & 194 & 0.37 \\
\hline 4041.00 & 0.6 & 4041.36 & Mn I & 5 & -0.36 \\
\hline & & 4041.64 & Fe II & 172 & -0.64 \\
\hline 4043.40 & 0.1 & 4044.00 & Fe II & 127 & -0.60 \\
\hline & & 4044.01 & FeII & 172 & -0.61 \\
\hline 4046.40 & 0.2 & 4045.81 & $\mathrm{Fe}_{\mathrm{I}}$ & 43 & 0.58 \\
\hline & & 4046.81 & Fe II & 126 & -0.41 \\
\hline 4047.80 & 0.4 & 4047.67 & $\alpha$ Cygni & & \\
\hline & & 4048.83 & Fe II & 172 & -1.03 \\
\hline 4050.60 & 0.1 & 4051.10 & VII & 32 & -0.50 \\
\hline
\end{tabular}


Table II (continued)

\begin{tabular}{|c|c|c|c|c|c|}
\hline \multirow[t]{2}{*}{$\lambda_{\text {obs }}$} & \multirow[t]{2}{*}{$W_{\lambda}$} & \multicolumn{3}{|c|}{ Identification } & \multirow[t]{2}{*}{$\delta$} \\
\hline & & $\lambda_{\text {lab }}$ & Ion & Mult. & \\
\hline 4050.60 & 0.1 & 4051.21 & $\mathrm{Fe}$ II & 172 & -0.61 \\
\hline 4052.60 & 0.1 & $\begin{array}{l}4051.97 \\
4052.63\end{array}$ & $\begin{array}{l}\text { Cr II } \\
v \text { Sag }\end{array}$ & 19 & 0.63 \\
\hline 4054.80 & 0.1 & $\begin{array}{l}4053.81 \\
4054.11 \\
4054.82\end{array}$ & $\begin{array}{l}\text { Ti II } \\
\text { Cr II } \\
v \text { Sag }\end{array}$ & $\begin{array}{l}87 \\
19\end{array}$ & $\begin{array}{l}0.99 \\
0.69\end{array}$ \\
\hline 4056.10 & 0.3 & $\begin{array}{l}4055.54 \\
4056.21\end{array}$ & $\begin{array}{l}\text { Mn I } \\
\text { Ti II }\end{array}$ & $\begin{array}{r}5 \\
11\end{array}$ & $\begin{array}{r}0.56 \\
-0.11\end{array}$ \\
\hline 4057.60 & 0.1 & 4057.46 & $\mathrm{Fe} I \mathrm{I}$ & 212 & 0.14 \\
\hline 4060.80 & 0.1 & $\begin{array}{l}4060.74 \\
4061.79\end{array}$ & $\begin{array}{l}v \text { Sag } \\
\text { Fe II }\end{array}$ & 189 & -0.99 \\
\hline 4063.80 & 0.4 & 4063.60 & $\mathrm{Fe}_{\mathrm{I}}$ & 43 & 0.20 \\
\hline 4064.40 & 0.1 & $\begin{array}{l}4064.35 \\
4065.07\end{array}$ & $\begin{array}{l}\text { TiII } \\
\text { VII }\end{array}$ & $\begin{array}{l}106 \\
215\end{array}$ & $\begin{array}{r}0.05 \\
-0.67\end{array}$ \\
\hline 4073.40 & 0.1 & 4072.60 & CriI & 26 & 0.80 \\
\hline 4078.60 & 1.1 & 4077.71 & Sr II & 1 & 0.89 \\
\hline 4080.40 & 0.3 & $\begin{array}{l}4079.85 \\
4080.44\end{array}$ & $\begin{array}{l}\mathrm{Fe} \mathrm{I} \\
v \mathrm{Sag}\end{array}$ & 359 & 0.55 \\
\hline 4080.90 & 0.2 & & & & \\
\hline 4081.70 & 0.2 & $\begin{array}{l}4081.42 \\
4082.30\end{array}$ & $\begin{array}{l}\text { Fe II } \\
\text { Cr II }\end{array}$ & $\begin{array}{l}188 \\
165\end{array}$ & $\begin{array}{r}0.28 \\
-0.60\end{array}$ \\
\hline 4087.20 & 0.2 & 4087.27 & Fe II & 28 & -0.07 \\
\hline 4088.70 & 0.1 & $\begin{array}{l}4088.75 \\
4088.90\end{array}$ & $\begin{array}{l}\text { Fe II } \\
\text { Cr II }\end{array}$ & $\begin{array}{l}39 \\
19\end{array}$ & $\begin{array}{l}-0.05 \\
-0.20\end{array}$ \\
\hline 4091.70 & 0.1 & & & & \\
\hline 4093.90 & 0.1 & 4093.90 & MgII & 29 & 0.00 \\
\hline 4094.50 & 0.1 & & & & \\
\hline 4095.70 & 0.2 & & & & \\
\hline 4098.50 & 0.1 & 4098.44 & Cr II & 165 & 0.06 \\
\hline 4100.70 & 0.1 & 4099.94 & $\mathrm{~N}_{\mathrm{I}}$ & 10 & 0.76 \\
\hline 4103.20 & 0.3 & & & & \\
\hline 4104.50 & 0.1 & 4104.18 & Fe II & 39 & 0.32 \\
\hline 4108.00 & 0.2 & 4107.49 & $\mathrm{Fe} I$ & 354 & 0.51 \\
\hline 4111.40 & 0.9 & $\begin{array}{l}4111.01 \\
4111.01\end{array}$ & $\begin{array}{l}\text { Cr II } \\
\text { Cr II }\end{array}$ & $\begin{array}{l}18 \\
26\end{array}$ & $\begin{array}{l}0.39 \\
0.39\end{array}$ \\
\hline 4111.90 & 0.4 & 4111.90 & FeII & 188 & 0.00 \\
\hline 4112.40 & 0.4 & 4112.59 & Cr II & 18 & 0.19 \\
\hline 4113.20 & 0.9 & 4113.24 & Cr II & 18 & -0.04 \\
\hline 4113.90 & 0.6 & 4114.00 & $\mathrm{NI}_{\mathrm{I}}$ & 10 & -0.10 \\
\hline 4115.20 & 0.4 & 4115.36 & $v \mathrm{Sag}$ & & \\
\hline 4116.90 & 0.2 & 4116.66 & CriI & 181 & 0.24 \\
\hline 4117.70 & 0.2 & 4118.00 & $v \mathrm{Sag}$ & & \\
\hline 4118.20 & 0.7 & 4118.55 & $\mathrm{Fe}_{\mathrm{I}}$ & 801 & -0.35 \\
\hline 4120.20 & 1.1 & 4119.53 & Fe II & 21 & 0.67 \\
\hline & & 4120.81 & $\mathrm{He} \mathrm{I}$ & 16 & -0.61 \\
\hline 4122.20 & 0.2 & 4122.64 & Fe II & 28 & -0.44 \\
\hline 4123.70 & 0.2 & 4123.75 & $\mathrm{Fe} I$ & 217 & -0.05 \\
\hline
\end{tabular}


Table II (continued)

\begin{tabular}{|c|c|c|c|c|c|}
\hline \multirow[t]{2}{*}{$\lambda_{\mathrm{obs}}$} & \multirow[t]{2}{*}{$W_{\lambda}$} & \multicolumn{3}{|c|}{ Identification } & \multirow[t]{2}{*}{$\delta$} \\
\hline & & $\lambda_{\text {lab }}$ & Ion & Mult. & \\
\hline 4123.70 & 0.2 & 4123.75 & $\mathrm{Fe}_{\mathrm{I}}$ & 422 & -0.05 \\
\hline 4124.80 & 0.2 & 4124.79 & $\mathrm{Fe}$ II & 22 & 0.01 \\
\hline 4125.70 & 0.1 & 4125.62 & $\mathrm{Fe}_{\mathrm{I}}$ & 1103 & 0.08 \\
\hline \multirow[t]{2}{*}{4129.50} & 0.3 & 4128.05 & SiII & 3 & 1.45 \\
\hline & & 4128.73 & Fe II & 27 & 0.77 \\
\hline 4130.70 & 0.1 & 4130.88 & Si II & 3 & -0.18 \\
\hline \multirow[t]{2}{*}{5132.00} & 0.1 & 4132.06 & $\mathrm{Fe}_{\mathrm{I}}$ & 43 & -0.06 \\
\hline & & 4132.41 & Cr II & 26 & -0.41 \\
\hline 4134.20 & 0.9 & 4134.34 & $\mathrm{Fe}_{\mathrm{I}}$ & 3 & -0.14 \\
\hline 4135.00 & 0.3 & 4134.68 & $\mathrm{Fe}_{\mathrm{I}}$ & 357 & 0.32 \\
\hline 4135.90 & 0.9 & 4136.30 & $v \mathrm{Sag}$ & & \\
\hline \multirow[t]{2}{*}{4136.70} & 0.8 & 4136.50 & $\mathrm{Fe} I$ & 694 & 0.20 \\
\hline & & 4137.00 & $\mathrm{Fe} I$ & 726 & -0.30 \\
\hline \multirow[t]{2}{*}{4138.70} & 0.8 & 4138.21 & Fe II & 150 & 0.49 \\
\hline & & 4138.40 & $\mathrm{Fe}$ II & 39 & 0.30 \\
\hline 4141.00 & 1.1 & 4141.25 & $v \mathrm{Sag}$ & & \\
\hline \multirow[t]{2}{*}{4142.70} & 1.4 & 4142.29 & $\mathrm{~S}_{\text {II }}$ & 44 & 0.41 \\
\hline & & 4143.42 & $\mathrm{Fe}_{\mathrm{I}}$ & 523 & -0.72 \\
\hline \multirow[t]{2}{*}{4145.50} & 0.4 & 4145.10 & S II & 44 & 0.40 \\
\hline & & 4145.77 & CrII & 162 & -0.27 \\
\hline 4146.30 & 0.3 & & & & \\
\hline \multirow[t]{2}{*}{4147.70} & 0.5 & 4147.26 & $\mathrm{Fe}$ II & 141 & 0.44 \\
\hline & & 4147.67 & $\mathrm{Fe}_{\mathrm{I}}$ & 42 & 0.03 \\
\hline \multirow[t]{3}{*}{4150.00} & 0.1 & 4149.22 & Zr II & 41 & 0.78 \\
\hline & & 4150.26 & $\mathrm{Fe} I$ & 695 & -0.26 \\
\hline & & 4151.00 & Cr II & 163 & -1.00 \\
\hline \multirow[t]{3}{*}{4152.10} & 0.1 & 4151.46 & $\mathrm{NI}_{\mathrm{I}}$ & 6 & 0.64 \\
\hline & & 4152.19 & Pleione & & \\
\hline & & 4152.98 & Fe II & 45 & -0.88 \\
\hline 4155.30 & 0.1 & 4154.81 & $\mathrm{Fe}_{\mathrm{I}}$ & 694 & 0.49 \\
\hline \multirow[t]{2}{*}{4159.30} & 0.2 & 4158.45 & Fe II & 12 & 0.85 \\
\hline & & 4159.45 & Al II & 71 & -0.15 \\
\hline \multirow[t]{3}{*}{4160.80} & 0.1 & 4160.62 & Fe II & 39 & 0.18 \\
\hline & & 4161.05 & Cr II & 162 & -0.25 \\
\hline & & 4161.52 & TiII & 21 & -0.72 \\
\hline 4164.50 & 0.2 & 4163.64 & TiII & 105 & 0.86 \\
\hline 4165.60 & 0.3 & & & & \\
\hline 4169.00 & 0.2 & 4168.66 & Fe II & 22 & 0.34 \\
\hline \multirow[t]{2}{*}{4172.80} & 0.1 & 4171.90 & TiII & 105 & 0.90 \\
\hline & & 4173.45 & Fe II & 27 & -0.65 \\
\hline \multirow[t]{3}{*}{4187.80} & 0.2 & 4187.04 & $\mathrm{Fe} I$ & 152 & 0.76 \\
\hline & & 4187.14 & Si II & 7.17 & 0.66 \\
\hline & & 4187.80 & $\mathrm{FeI}$ & 152 & 0.00 \\
\hline 4208.10 & 0.2 & 4207.35 & CrII & 26 & 0.75 \\
\hline \multirow[t]{3}{*}{4209.30} & 0.2 & 4208.99 & Zr II & 41 & 0.31 \\
\hline & & 4209.02 & CrII & 162 & 0.28 \\
\hline & & 4210.35 & $\mathrm{Fe} I$ & 152 & -1.05 \\
\hline 4211.40 & 0.2 & 4211.88 & Zr II & 15 & -0.48 \\
\hline
\end{tabular}


Table II (continued)

\begin{tabular}{|c|c|c|c|c|c|}
\hline \multirow{2}{*}{$\lambda_{\text {obs }}$} & \multirow[t]{2}{*}{$W_{\lambda}$} & \multicolumn{3}{|c|}{ Identification } & \multirow[t]{2}{*}{$\delta$} \\
\hline & & $\lambda_{\text {lab }}$ & Ion & Mult. & \\
\hline 4211.40 & 0.2 & 4212.10 & Fe II & 21 & -0.70 \\
\hline 4216.10 & 0.2 & 4215.52 & Sr II & 1 & 0.58 \\
\hline 4217.40 & 0.1 & 4217.55 & $\mathrm{Fe}_{\mathrm{I}}$ & 693 & -0.15 \\
\hline 4222.10 & 0.1 & 4222.22 & $\mathrm{Fe}_{\mathrm{I}}$ & 152 & -0.12 \\
\hline 4224.10 & 0.1 & 4223.84 & v Sag & & \\
\hline 4225.30 & 0.1 & 4224.85 & CriI & 162 & 0.45 \\
\hline \multirow[t]{2}{*}{4228.10} & 0.1 & 4227.34 & TiII & 33 & 0.76 \\
\hline & & 4227.43 & $\mathrm{Fe}_{\mathrm{I}}$ & 693 & 0.67 \\
\hline 4232.10 & 0.2 & 4233.17 & Fe II & 27 & -1.07 \\
\hline 4244.20 & 0.2 & 4244.80 & Ni II & 9 & -0.60 \\
\hline \multirow[t]{2}{*}{4247.10} & 0.1 & 4246.41 & Cr II & 31 & 0.69 \\
\hline & & 4246.83 & Sc II & 7 & 0.27 \\
\hline \multirow[t]{2}{*}{4248.60} & 0.2 & 4248.03 & v Sag & & \\
\hline & & 4248.23 & $\mathrm{FeI}$ & 482 & 0.37 \\
\hline \multirow[t]{3}{*}{4250.60} & 0.1 & 4250.12 & $\mathrm{Fe} I$ & 152 & 0.48 \\
\hline & & 4250.79 & $\mathrm{Fe} I$ & 42 & -0.19 \\
\hline & & 4251.49 & Fe II & 12 & -0.89 \\
\hline \multirow[t]{2}{*}{4254.90} & 0.1 & 4254.35 & $\mathrm{CrI}$ & 1 & 0.55 \\
\hline & & 4254.70 & $\mathrm{~N}_{\mathrm{I}}$ & 4 & 0.20 \\
\hline 4257.60 & 0.1 & 4258.15 & $\mathrm{Fe} I \mathrm{II}$ & 28 & -0.55 \\
\hline 4261.60 & 0.2 & 4261.92 & Cr II & 31 & -0.32 \\
\hline 4262.60 & 0.1 & 4263.20 & $\alpha$ Cygni & & \\
\hline 4269.70 & 0.2 & 4269.28 & CrII & 31 & 0.42 \\
\hline 4285.40 & 0.3 & 4285.41 & $\mathrm{Fe} I$ & 597 & -0.01 \\
\hline \multirow[t]{2}{*}{4286.70} & 0.2 & 4286.30 & V II & 23 & 0.40 \\
\hline & & 4286.31 & Fe II & & 0.39 \\
\hline 4288.10 & 0.3 & 4287.89 & TiII & 20 & 0.21 \\
\hline
\end{tabular}

For lines shortward of $\lambda 3716$ or longward of $\lambda 4290$, the available data are very poor, positions being accurate to only about $3 \AA$. Consequently, we have not attempted to identify these highly blended lines, but instead we predict the expected spectrum in the visible region outside of $\lambda \lambda 3716-4290$ on the basis of our identifications of Table II.

HeI. Many lines should be present.

Ti II, Cr II, Fe I, and FeII. Many multiplets which arise from low-lying terms in these ions should be present, especially those shown on the Grotrian diagrams of Moore and Merrill (1968). This prediction is based on the assumption that there is no significant change in the spectrum in the region $\lambda \lambda 3716-4290$. It is known that shell stars change with time. Should a significant change have occurred in the spectrum in the region $\lambda \lambda 3716-4290$ when future observations are carried out, obviously a corresponding modification will have to be made in the prediction.

It would be most desirable to obtain the spectrum of $0237-233$ over a wide wavelength interval (3200-5000 $)$ at a high resolution $c / R \simeq 10 \mathrm{~km} \mathrm{~s}^{-1}$ ) with instrumentation which can detect weaker lines. 


\section{The Discontinuity in the Ultraviolet}

Bechtold et al. (1984) have measured the continuum energy distribution of $0237-233$ with the International Ultraviolet Explorer and the Palomar $5 \mathrm{~m}$ multichannel spectrophotometer. A discontinuity was found in the ultraviolet and these authors state that it occurs at $2350 \pm 10 \AA$. On the other hand, from the PLS model, for an atmosphere rich in He I, we expect a discontinuity at $\lambda 2600$ (the ionization limit from the $1 s 2 s^{3} S$ state). To examine the problem carefully, we show in Figure 1, the observed data reported by Bechtold et al. (1984). The positions of $\lambda 2350$ and $\lambda 2600$ are indicated. There is an observed point at $2614 \AA$, but as shown in the figure, it has very large error bars. Allowing for this large uncertainty, the observed data are consistent with a discontinuity at $\lambda 2600$. We predict that more accurate observations will confirm the expected discontinuity at $\lambda 2600$.

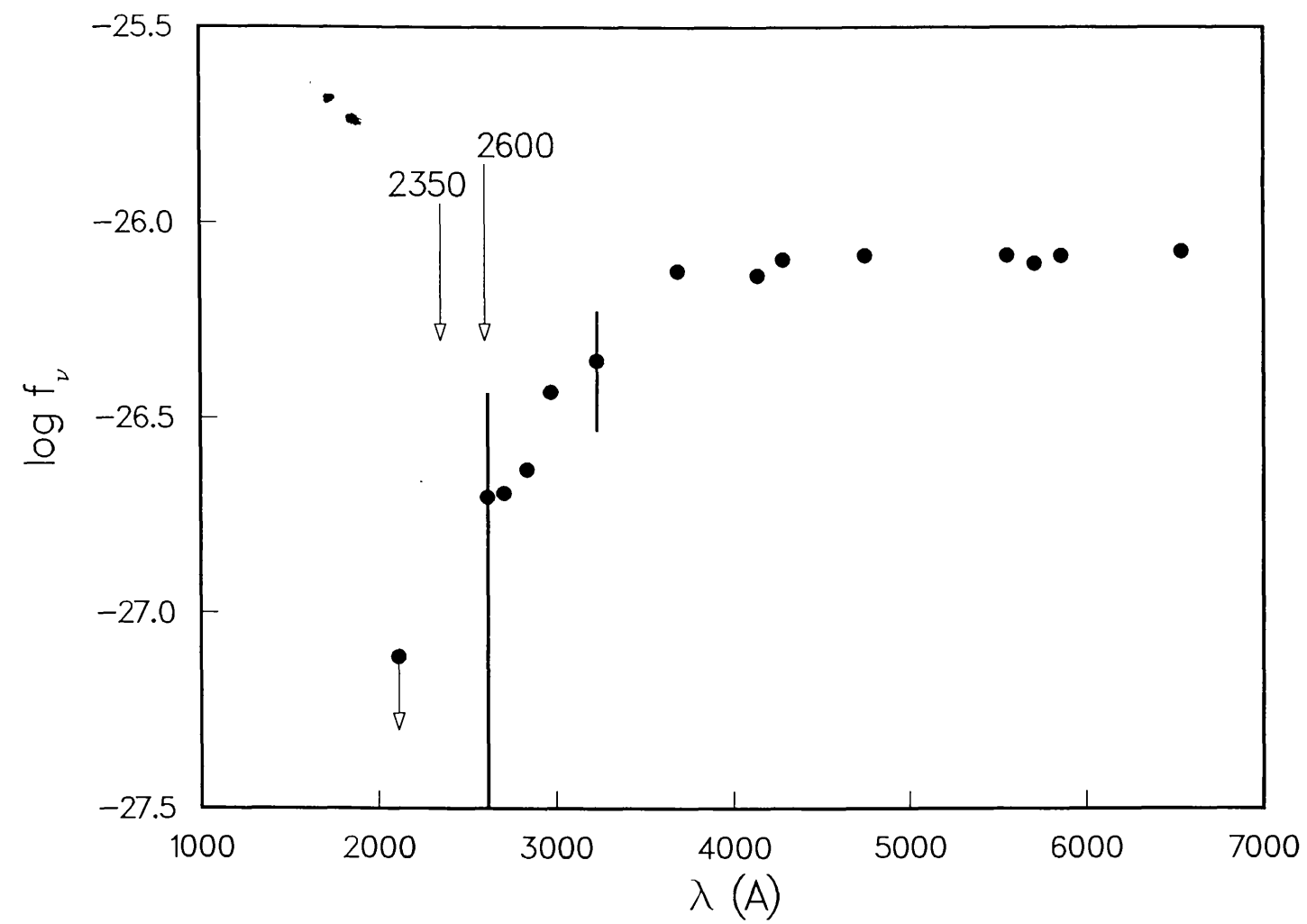

Fig. 1. Continuum spectral energy distribution of $0237-233$.

\section{Similarities with Upsilon Sagittarii}

A comparison of the absorption lines in the spectrum of $0237-233$ in the interval $\lambda \lambda 3716-4290$ with the spectrum of the star $v$ Sgr shows that many of the identifications are common. Not only that, most of the unidentified lines in 0237-233 are common with unidentified lines in the spectrum of $v$ Sgr. Clearly a comparison between other spectral properties is of interest. 
First we briefly summarize some of the important data about $v$ Sgr. The rich spectrum of $v$ Sagittarii (HD 181615; mag. 4.6; spect. B8-A2 Ia) has been investigated by a number of workers (Plaskett, 1928; Greenstein, 1940, 1943; Greenstein and Merrill, 1946; Greenstein and Adams, 1947). A strong helium spectrum is superposed on a complex metallic spectrum. Hydrogen lines are unusually weak. Emission lines are also present in the red and infra-red regions - $\mathrm{H} \alpha$ and those of $\mathrm{CaII}$ and [CaII], FeII and possibly [FeII]. It is believed there is an extended nebulous envelope around the star. Hack and Pasinetti (1963) found that the star is deficient in hydrogen and estimated $n(\mathrm{H}) / n(\mathrm{He})=0.025$. A more recent analysis by Schoenberner and Drilling (1983) indicates a much lower hydrogen abundance, i.e., $n(\mathrm{H}) / n(\mathrm{He})<0.0005$. The observed spectrum is that of a single star. This star is a member of a binary system (Wilson, 1914). In the visible region no spectroscopic evidence has been detected of the other member, but in the ultraviolet region, spectroscopic evidence has been obtained recently (Parthasarathy et al., 1986).

Next we compare $0237-233$ with $v$ Sgr.

(1) The absorption line spectra of the two objects are rather similar. Both objects are strongly deficient in hydrogen.

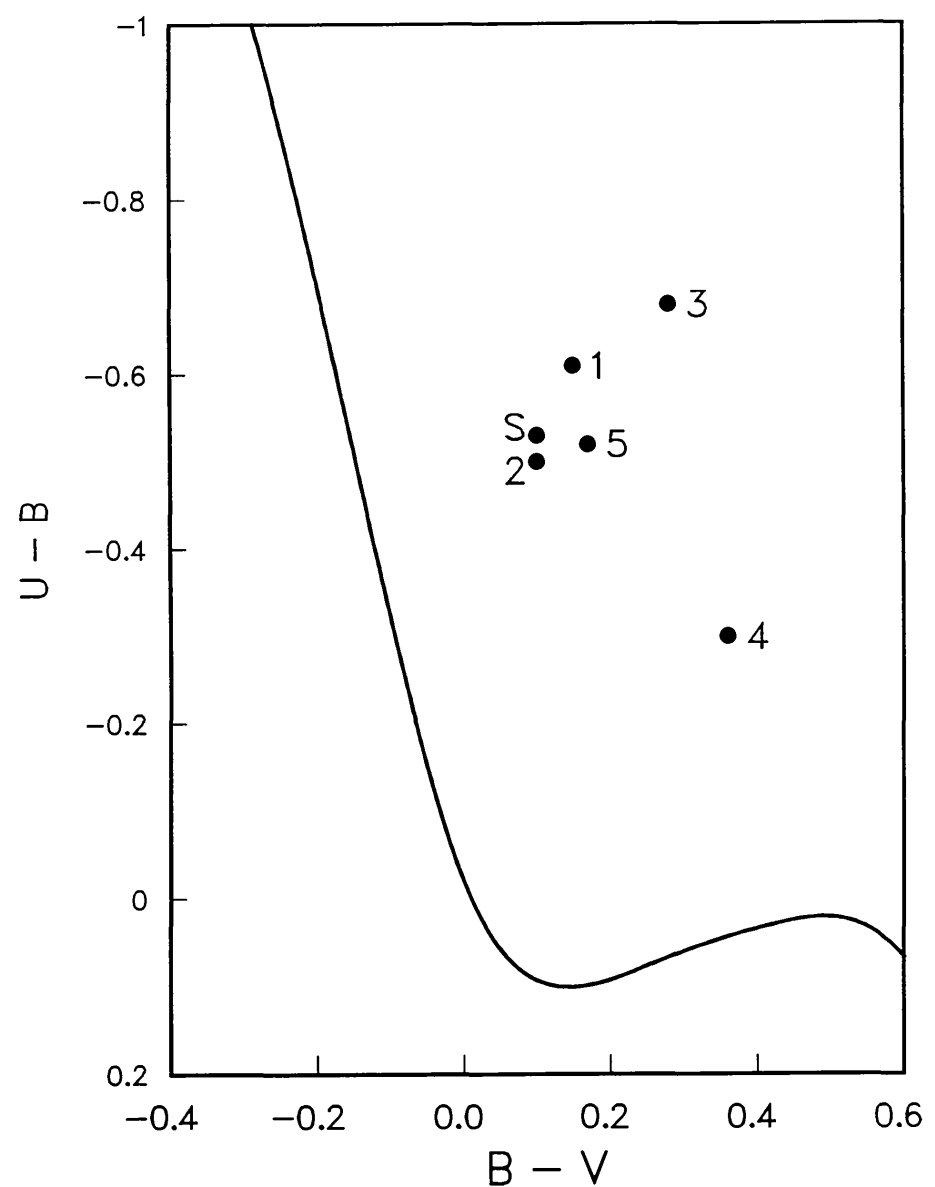

Fig. 2. $(U-B)$ versus $(B-V)$ plot. Key to points $1: 0237-233,2: 0851+197,3: 1225+317,4: 1246-057$, 5: $1448-232, \mathrm{~S}: v$ Sgr. 
(2) Both objects show emission lines in their spectra, but the nature of the emission lines in the two is different. The emission lines in $0237-233$ are broad, while those in $v$ Sgr are relatively sharp. $v$ Sgr shows several emission lines in the red region (Greenstein and Merrill, 1946). It would be of interest to investigate if there is any emission at those wavelengths in $0237-233$.

(3) In Figure 2 we show the positions of $0237-233$ and $v$ Sgr on the two-colour $(U-B)$ versus $(B-V)$ plot. The positions of a few other quasars in Table I for which data are available and also shown. $0237-233$ and $v$ Sgr are seen to be very close on this plot.

(4) Both objects show large infrared excesses. Infrared photometry of $v$ Sgr has been carried out by Lee and Nariai (1967), Woolf (1973), and by Treffers et al. (1976), and that of $0237-233$ by Hyland and Allen (1982). In Figure 3 we compare the colours of $0237-233$ with those of $v$ Sgr. It will be noted that both objects are very red at long wavelengths and the spectral energy distributions of the two are very similar. Two hypotheses have been advanced to explain the infra-red excess in $v$ Sgr. A late-type secondary or the circumstellar envelope. Parthasarathy et al. (1986) have found that the companion is a hot star (late $\mathrm{O}$ or early B-type). Thus the second possibility seems most likely (see also Treffers et al., 1976). In the case of $0237-233$, it is a natural consequence of our theory that the ejected material from the star will form a circumstellar envelope and in due course some of it will condense to form dust, which will lead to infrared

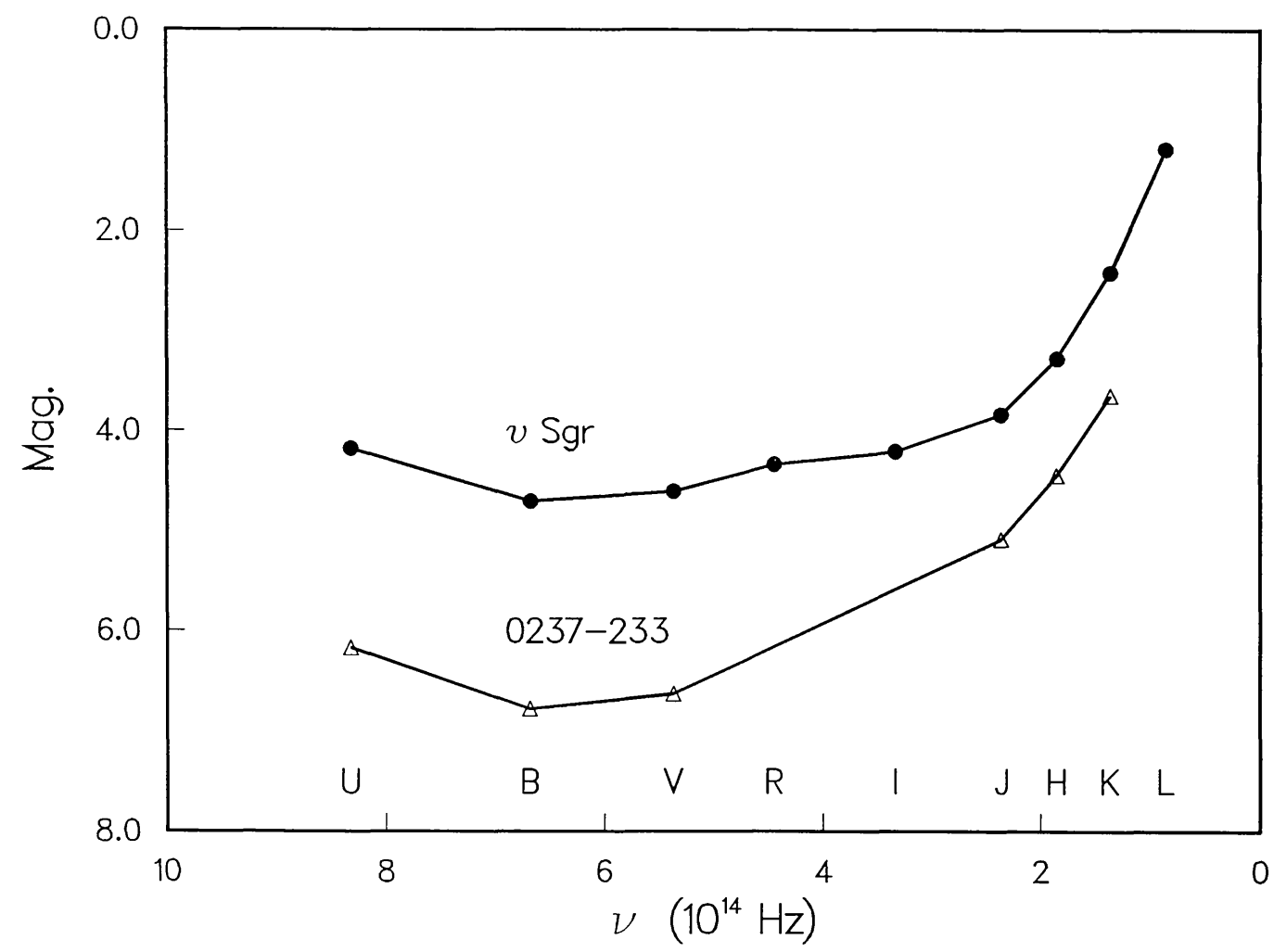

Fig. 3. The colours of $v \mathrm{Sgr}$ and $0237-233$. The data points for $v$ Sgr are from Lee and Nariai (1967) and those for 0237 - 233, from Hewitt and Burbidge (1987) and Hyland and Allen (1983). To accomodate the two objects on the same plot, the magnitudes for $0237-233$ have been increased by 10 . 
excess. $v$ Sgr shows a $10 \mu$ emission feature (Treffers et al., 1976), which is similar to the $10 \mu$ emission feature found around oxygen-rich stars and interpreted as being due to silicates. Irrespective of its interpretation it would be of interest to investigate if $0237-233$ also shows the $10 \mu$ emission feature.

(5) The positions of $0237-233$ and $v$ Sgr on the $(J-H)$ versus $(H-K)$ plot are shown in Figure 4. Two other quasars in Table I, for which data are available, are also shown. In judging the proximity of the points, it should be kept in mind that we have shown here only a small portion of the usual $(J-H)$ versus $(H-K)$ diagram for quasars in which both axes run from 0 to 1.5 (Hyland and Allen, 1982). Again, here also, as in Figure 2, $0237-233$ and $v$ Sgr are seen to be in close proximity.

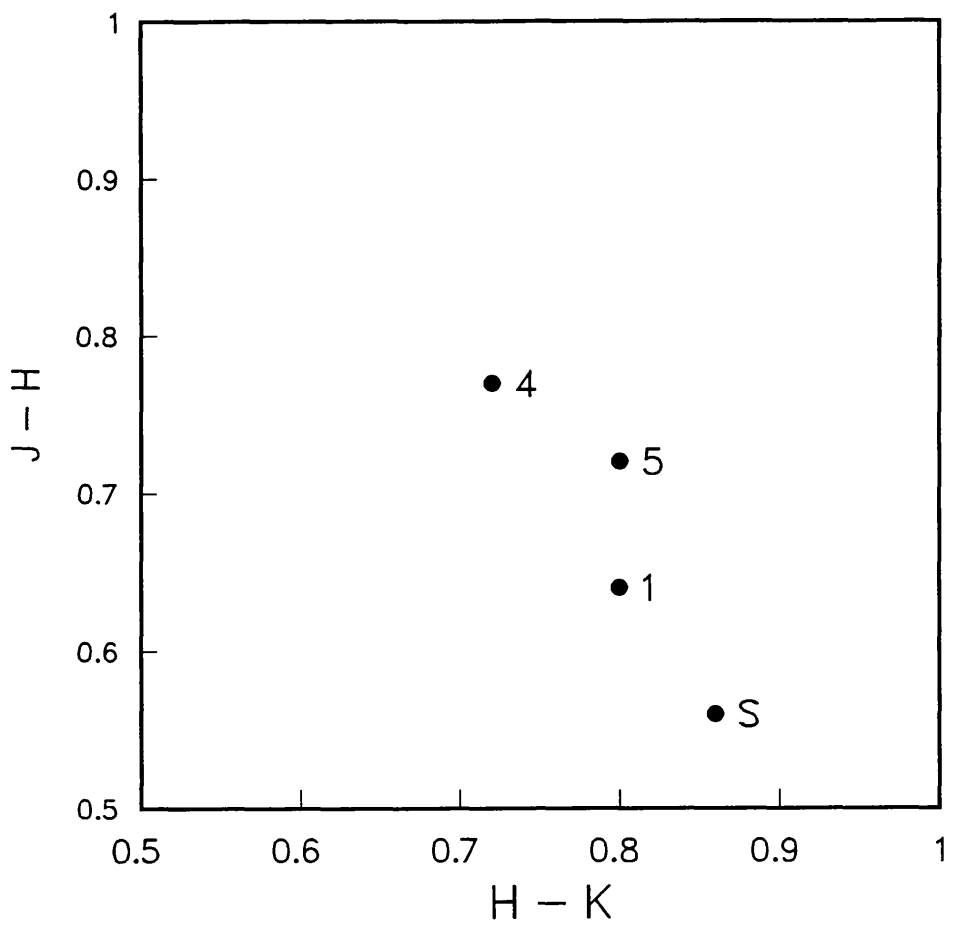

Fig. 4. The $(J-H)$ versus $(H-K)$ diagram for 3 quasars and $v$ Sgr. Symbols have the same meaning as in Figure 2.

(6) The polarization in the optical region in both cases is small $-\sim 0.25 \%$ for $0237-233$ (Moore and Stockman, 1984) and $\leq 1 \%$ for $v$ Sgr (Coyne and Gehrels, 1967).

It is not implied or suggested that the absolute magnitudes of $0237-233$ and $v$ Sgr (Rao and Venugopal, 1985) might be similar. Rather we think that $0237-233$ is an intrinsically fainter object (Varshni, 1977a) than $v$ Sgr. The spectral similarities between the two are reminiscent of the spectral similarities between classical Wolf-Rayet stars and some planetary nuclei. We suspect that $0237-233$ has some relation with hydrogendeficient planetary nuclei (Mendez et al., 1986).

$v$ Sgr is known to be a binary. Thus naturally the question arises if $0237-233 \mathrm{might}$ also be a binary. The historical light curve of $0237-233$ has been summarized by 
Angione (1973). The available data are rather patchy but are suggestive of an eclipsing binary nature.

\section{Predictions}

We summarize our predictions.

(1) In the optical region outside of $\lambda \lambda 3716$ - 4290, many lines due to He I, TiII, Cr II, Fe I, and Fe II should be observable, especially those arising from low-lying terms in the case of TiII, CrII, FeI, and Fe II.

(2) Narrow-band spectrophotometry would show that the discontinuity in the ultraviolet is close to 22600 . Further if any discontinuity is observed in the ultraviolet in any of the other quasars listed in Table I, it is expected to occur either at $\lambda 2600$ or at $\lambda 3122$ (the ionization limit from the $1 s 2 s{ }^{1} S$ state of He I).

The crying need in quasar spectral studies is for better resolution, greater accuracy and the ability to detect weaker lines.

\section{References}

Angione, R. J.: 1973, Astron. J. 78, 353.

Arp, H. C., Bolton, J. G., and Kinman, T.: 1967, Astrophys. J. 147, 841.

Bahcall, J. N., Greenstein, J. L., and Sargent, W. L. W.: 1968, Astrophys. J. 153, 689.

Bechtold, J., Green, R. F., Weymann, R. J., Schmidt, M., Estabrook, F. B., Sherman, R. D., Wahlguist, H. D., and Heckman, T. M.: 1984, Astrophys. J. 281, 76.

Boksenberg, A. and Sargent, W. L. W.: 1975, Astrophys. J. 198, 31.

Boroson, T., Sargent, W. L. W., Boksenberg, A., and Carswell, R. F.: 1978, Astrophys. J. $220,772$.

Burbidge, E. M.: 1967, Astrophys. J. 147, 845.

Burbidge, E. M., Lynds, C. R., and Stockton, A. N.: 1968, Astrophys. J. 152, 1077.

Chaffee, F. H., Weymann, R. J., Latham, D. W., and Strittmatter, P. A.: 1983, Astrophys. J. $267,12$.

Chen, J.-S., Morton, D. C., Peterson, B. A., Wright, A. E., and Jauncey, D. L.: 1983, Kexue Tongbao 28, 1075.

Chen, J.-S., Morton, D. C., Peterson, B. A., Wright, A. E., and Jauncey, D. L.: 1984, Proc. Astron. Soc. Australia 5, 355.

Coyne, G. V. and Gehrels, T.: 1967, Astron. J. 72, 887.

Greenstein, J. L.: 1940, Astrophys. J. 91, 438.

Greenstein, J. L.: 1943, Astrophys. J. 97, 252.

Greenstein, J. L.: 1984, Ann. Rev. Astron. Astrophys. 22, 1.

Greenstein, J. L. and Adams, W. S.: 1947, Astrophys. J. 106, 339.

Greenstein, J. L. and Merrill, P. W.: 1946, Astrophys. J. 104, 177.

Greenstein, J. L. and Schmidt, M.: 1967, Astrophys. J. 148, L13.

Grueff, G.: 1969, Astrophys. Letters 4, 141.

Hack, M. and Pasinetti, L.: 1963, Contr. Oss. Astr. Milano- Merate, No. 215.

Hewitt, A. and Burbidge, G.: 1987, Astrophys. J. Suppl. 63, 1.

Hyland, A. R. and Allen, D. A.: 1982, Monthly Notices Roy. Astron. Soc. 199, 943.

Jauncey, D. L., Batty, M. J., Wright, A. E., Peterson, B. A., and Savage, A.: 1984, Astrophys. J. $286,498$. Lee, T. A. and Nariai, K.: 1967, Astrophys. J. 149, L93.

MacAlpine, G. M. and Lewis, D. W.: 1978, Astrophys. J. Suppl. 36, 587.

Mendez, R. H., Miguel, C. H., Heber, U., and Kudritzki, R. P.: 1986, in K. Hunger, D. Schonberner, and N. K. Rao (eds.), Hydrogen Deficient Stars and Related Objects, D. Reidel Publ. Co., Dordrecht, Holland, p. 323.

Moore. C. E. and Merrill, P. W.: 1968, Partial Grotrian Diagrams of Astrophysical Interest, NSRDS-NBS 23, U.S. Government Printing Office, Washington, D.C. 
Moore, R. L. and Stockman, H. S.: 1984, Astrophys. J. 279, 465.

Osmer, P. S. and Smith, M. G.: 1977, Astrophys. J. 213, 607.

Parthasarathy, M., Cornachin, M., and Hack, M.: 1986, Astron. Astrophys. 166, 237.

Plaskett, J. S.: 1928, Publ. Dominion Astrophys. Obs. 4, 103.

Rao, N. K. and Venugopal, V. R.: 1985, J. Astrophys. Astron. 6, 101.

Savage, A., Browne, I. W. A., and Bolton, J. G.: 1976, Monthly Notices Roy. Astron. Soc. 177, 77P.

Savage, A., Clowes, R. G., Cannon, R. D., Cheung, K., Smith, M. G., Boksenberg, A., and Wall, J. V.: 1985, Monthly Notices Roy. Astron. Soc. 213, 485.

Savage, A. and Wright, A. E.: 1981, Monthly Notices Roy. Astron. Soc. 196, 927.

Schmidt, M.: 1974, Astrophys. J. 193, 509.

Schoenberner, D. and Drilling, J. S.: 1983, Astrophys. J. 268, 225.

Shaver, P. A., Boksenberg, A., and Robertson, J. G.: 1982, Astrophys. J. 261, L7.

Treffers, R., Woolf, N. J., Fink, U., and Larson, H. P.: 1976, Astrophys. J. 207, 680.

Ulrich, M.-H.: 1976, Astrophys. J. 207, L73.

Varshalovich, D. A. and Levshakov, S. A.: 1981, Soviet Astron. Letters 7, 113.

Varshni, Y. P.: 1973, Bull. Am. Phys. Soc. 18, 1384.

Varshni, Y. P.: 1974, Bull. Am. Astron. Soc. 6, 213, 308.

Varshni, Y. P.: 1975, Astrophys. Space Sci. 37, L1.

Varshni, Y. P.: 1976, Astrophys. Space Sci. 43, 3.

Varshni, Y. P.: 1977a, Astrophys. Space Sci. 46, 443.

Varshni, Y. P.: 1977b, J. Roy. Astron. Soc. Canada 71, 403.

Varshni, Y. P.: 1978, in S. Fujita (ed.), The Ta-You Wu Festschrift: Science of Matter, Gordon and Breach, New York, p. 285.

Varshni, Y. P.: 1979, Phys. Canada 35, 11.

Varshni, Y. P.: 1981, Astrophys. Space Sci. 74, 3.

Varshni, Y. P.: 1982, Spec. Sci. Tech. 5, 521.

Varshni, Y. P.: 1983, Soviet Astron. Letters 9, 368.

Varshni, Y. P.: 1985, Astrophys. Space Sci. 117, 337.

Varshni, Y. P.: 1988a, Bull. Am. Astron. Soc. 20, 733.

Varshni, Y. P.: 1988b, Bull. Am. Phys. Soc. 33, 1184.

Varshni, Y. P. and Lam, C. S.: 1976, Astrophys. Space Sci. 45, 87.

Varshni, Y. P. and Nasser, R. M.: 1986, Astrophys. Space Sci. 125, 341.

Walsh, D., Wills, B. J., and Wills, D.: 1979, Monthly Notices Roy. Astron. Soc. 189, 667.

Wilson, R. E.: 1914, Lick Obs. Bull. 8, 134.

Woolf, N. J.: 1973, Astrophys. J. 185, 229.

York, D. G., Green, R. F., Bechtold, J., and Chaffee, F. H., Jr.: 1984, Astrophys. J. 280 , L1. 